\title{
Low Amino Acid Score of Breakfast is Associated with the Incidence of Cognitive Impairment in Older Japanese Adults: A Community-Based Longitudinal Study
}

\author{
K. Kinoshita ${ }^{1,2}$, R. Otsuka ${ }^{1}$, M. Takada ${ }^{3}$, M. Tsukamoto-Yasui ${ }^{4}$, Y. Nishita ${ }^{5}$, C. Tange ${ }^{1}$, M. Tomida ${ }^{1}$, H. Jinzu ${ }^{6}$, \\ H. Shimokata ${ }^{1,7}$, M. Kuzuya ${ }^{2}$, A. Imaizumi ${ }^{3}$, H. Arai ${ }^{8}$
}

1. Section of NILS-LSA, Center for Gerontology and Social Science, National Center for Geriatrics and Gerontology, Aichi, Japan; 2. Department of Community Healthcare and Geriatrics, Nagoya University Graduate School of Medicine, Aichi, Japan; 3. Research Institute for Bioscience Products \& Fine Chemicals, Ajinomoto Co., Inc., Kanagawa, Japan; 4. R \& B Planning Department, Ajinomoto Co., Inc., Tokyo, Japan; 5. Department of Epidemiology of Aging, Center for Gerontology and Social Science, National Center for Geriatrics and Gerontology, Aichi, Japan; 6. Institute of Food Sciences and Technologies, Ajinomoto Co., Inc., Kanagawa, Japan; 7. Graduate School of Nutritional Sciences, Nagoya University of Arts and Sciences, Aichi, Japan; 8. National Center for Geriatrics and Gerontology, Aichi, Japan

Corresponding Author: Rei Otsuka, Section of NILS-LSA, Center for Gerontology and Social Science, National Center for Geriatrics and Gerontology, 7-430 Morioka, Obu, Aichi 474-8511, Japan, E-mail: otsuka@ncgg.go.jp; Tel: +81-562-46-2311; Fax: +81-562-48-2373

\begin{abstract}
BACKGROUND: The protein digestibility-corrected amino acid score (PDCAAS) represents the degree of utilizable dietary protein, namely the protein quality. The PDCAAS of a diet is required to be evaluated on a meal-by-meal basis, as food digestion and absorption occur with each meal intake. Although a positive association between protein intake and cognitive function has been reported, no study has investigated the association between PDCAAS of a diet and cognitive function.

Objectives: To investigate the relationship between PDCAAS of a diet and cognitive impairment in older adults.
\end{abstract}

DESIGN: Longitudinal epidemiological study.

SETTING: Community-based setting.

PARTICIPANTS: We analyzed 541 community-dwellers who participated in both baseline and follow-up survey. They were 60-83 years of age without cognitive impairment at baseline.

MEASUREMENTS: Cognitive impairment was defined as a Mini-Mental State Examination (MMSE) score $\leq 27$. Individual PDCAASs were calculated for each of three regular meals from the 3-day dietary records at baseline. Participants were classified into two groups according to the sex-specific tertiles (T1-T3) of the PDCAAS for each meal (i.e., T1 as the low score group and T2-T3 as the medium and high score group). The dependent variable was cognitive impairment observed after 4 years, and the explanatory variables were the PDCAAS groups for each meal (the medium and high group as the reference) and covariates (sex, age, body mass index, education, depressive symptoms, medical history, protein intake at each meal, and the MMSE score at baseline). Multivariable logistic regression analysis was performed to evaluate the low PDCAAS group for cognitive impairment after 4 years.

RESULTS: A significant association was observed only between a low PDCAAS of breakfast and the incidence of cognitive impairment (the adjusted odds ratios [95\% confidence intervals] of low PDCAAS for cognitive impairment for breakfast, lunch, and dinner were 1.58 [1.00-2.50], 0.85 [0.54-1.34], and 1.08 [0.711.65], respectively).

CONCLUSION: A lower PDCAAS of breakfast, i.e., a diet with poor quality of protein, was associated with the incidence of cognitive impairment in older adults of the community.
Key words: Protein quality, dietary amino acid, three regular meals, cognition, aging.

\section{Introduction}

$\Lambda$ $s$ aging is progressing rapidly and the prevalence of dementia such as Alzheimer's disease is increasing worldwide, measures to delay or to prevent cognitive decline is extremely important for public health. In this regard, expectations of the nutritional approaches for delaying the occurrence of dementia are growing (1).

Several amino acids from dietary proteins act as neurotransmitter precursors; studies have shown a positive association between dietary protein and cognitive function $(2,3)$. van de Rest et al. (3) indicated that the effect of protein intake on cognitive function may be attributed to the intake of specific essential amino acids such as tryptophan. Dietary protein, however, comprises several types of amino acids. The bioavailability of dietary protein is measured by protein quality; it refers to the proportion of amino acids that can be absorbed from the diet and utilized (4). Protein quality is evaluated by the amino acid score, wherein the capacity of a diet to satisfy the metabolic demand for amino acids and nitrogen is assessed (4).

The methods for evaluating protein quality have been considered since 1955 (5). In 1991, the Joint Food and Agriculture Organization (FAO) of the United Nations and World Health Organization (WHO) Expert Consultation on Protein Quality Evaluation published the protein digestibility-corrected amino acid score (PDCAAS) for evaluating protein quality (6). Currently, the digestible indispensable amino acid score (DIAAS) is recommended as successor to the PDCAAS (7). The DIAAS was developed to overcome limitations of the 
PDCAAS; however, we lack data on ileal digestibility for commonly consumed human foods, needed for the calculation of DIAAS (7). Thus, the PDCAAS is currently a better method to evaluate protein quality in diets $(8,9)$.

Although older adults often present with ageassociated reduction in food-intake, protein requirements increase in old age $(10,11)$. Optimal intake of protein is especially important for older people. Therefore, it is meaningful to verify the role of dietary protein quality in maintaining cognitive function in older adults by evaluating the PDCAAS of the diet. However, to the best of our knowledge, no study has investigated the association between the PDCAAS of diet and cognitive function in older adults. Further, PDCAAS of diets may be required to be evaluated on a meal-by-meal basis and not just for the total intake, as food digestion and absorption occur with each meal intake. In recent years, the importance of meal time for breakfast, lunch, and dinner is being re-evaluated for human health, and the focus is not only on the effects of "what" we consume but also on "when" we consume a meal (12).

Therefore, we aimed to investigate the longitudinal association between the PDCAAS, calculated for breakfast, lunch, dinner, and the total daily intake, and incidence of cognitive impairment in communitydwelling older adults.

\section{Methods}

\section{Study design and participants}

This study was based on the National Institute for Longevity Sciences-Longitudinal Study of Aging (NILSLSA) (13). NILS-LSA participants were aged 40-79 years at first participation and were recruited using the methods of stratified random sampling by age and sex from the residents of Obu city and Higashiura town in Aichi Prefecture, Japan.

In this study, we set the third wave of the NILS-LSA (May 2002 to May 2004, n=2,378) as a baseline study cohort. The first wave (November 1997 to April 2000) was excluded owing to a lack of cognitive function measurements. The second wave (April 2000 to May 2002) was affected by the outbreak of the British bovine spongiform encephalopathy that led to a reduction of meat consumption in some countries, including Japan (14). As meat is the main source of amino acids, we set the third wave as the baseline in this study.

At baseline, 1,202 participants aged $\geq 60$ years were included because the NILS-LSA project assessed the Mini-Mental State Examination (MMSE) for only this age group; 849 of them participated in the follow-up study-the fifth wave- between July 2006 and July 2008. We excluded participants who had cognitive impairment at baseline (as defined by MMSE $\leq 27, \mathrm{n}=251$ ) (15-17), did not have their cognitive function assessed $(n=11)$, had not submitted dietary records $(n=27)$, had skipped at least one of the three regular meals (i.e., $0 \mathrm{kcal}, \mathrm{n}=2$ ), or had missing data for covariates $(n=17)$. Finally, 541 participants were analyzed (mean age \pm standard deviation [SD], 68.2 \pm 5.7 years; males, $n=256$; females, $n=285$ ) in this study (Figure 1).

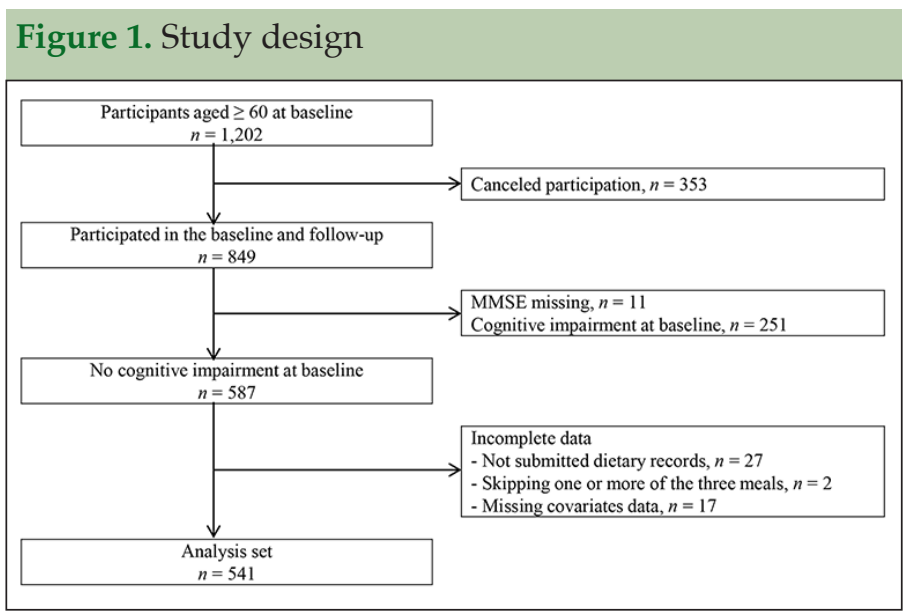

Participants who met the age requirements for the baseline study and participated in the follow-up study were included. We excluded the participants who displayed already cognitive impairment at baseline, did not have their cognitive function assessed, had not submitted dietary records, had skipped one or more of the three meals, or had missing data for covariates. Finally, data from 541 participants were analyzed in this study. MMSE, Mini-Mental State Examination

All participants provided written informed consent before the commencement of the study. This study was conducted in accordance with the guidelines of the Declaration of Helsinki; all procedures were approved by the Ethics Committee of Human Research of the National Center for Geriatrics and Gerontology, Japan (No. 1115-3) and Ethics Committee of Ajinomoto Co., Inc. (No. 2017032).

\section{Dietary assessment}

Participants assessed their baseline average dietary intake at home, with a continuous 3-day dietary recordtwo weekdays and one day on the weekend (18). The participants weighed each food item with a kitchen scale before cooking (Sekisui Jushi, Tokyo, Japan) and took photos of their meals before and after eating, using a disposable camera (Fuji Film, Tokyo, Japan). The dietary record comprised nine sheets (three regular meals for three days), and all participants recorded the entries in chronological order. Averages of dietary intake for each meal were calculated based on the Standard Tables of Food Composition in Japan (19).

\section{Calculation of the amino acid score}

PDCAASs were calculated for each meal (i.e., breakfast, lunch, and dinner) and the total daily meal. These were calculated based on the FAO/WHO/United Nations University (UNU) Expert Consultation as follows: PDCAAS = digestibility $\times$ amino acid score (6). For amino 
acid score calculation, the nine essential amino acid requirement patterns were referred to, as proposed by FAO/WHO/UNU $(5,6)$. The amino acid score is defined as the lowest ratio of amino acids in dietary protein, calculated by comparing the nine essential amino acids to their requirement patterns (6). Amino acid scores were calculated as follows: Amino acid score $(\%)=$ amount of amino acid (mg) in $1 \mathrm{~g}$ protein / amount of amino acid $(\mathrm{mg})$ in requirement pattern $\times 100$ (6). If the amino acid score of a diet exceeded $100 \%$, it was rounded down to $100 \%$ (6). Our study investigated the PDCAAS of a diet using the existing epidemiological data. Generally, people consume a diet comprising mixed foods. Although the $\mathrm{WHO} / \mathrm{FAO} / \mathrm{UNU}$ report demonstrated the true protein digestibility of some mixed diets (e.g., American diet, 96\%; Filipino diet, 88\%; and Chinese diet, 96\%), it did not include the Japanese mixed diet $(4,6)$. Thus, individual protein digestibility of the diet in this study was set at $90 \%$, based on Japanese literature on the Japanese mixed $\operatorname{diet}(20)$.

\section{Assessment of cognitive function}

A trained psychologist or psychology graduate students assessed cognitive function using the Japanese version of the MMSE $(21,22)$, at both baseline and follow-up. The MMSE score ranges from 0 to 30 with higher scores indicating better cognitive function (23). Generally, an MMSE score of $\leq 23$ is used as a cut-off for suspected dementia; however, only 33 participants had an MMSE $\leq 23$ at follow-up in the present study. Thus, the number of cases was too small to analyze. Meanwhile, people with MMSE scores of $\leq 27$ are suspected of mild cognitive impairment, as suggested in some studies (1517). Preventing cognitive decline at an early stage is important. Therefore, MMSE score of $\leq 27$ was used as the cut-off for cognitive impairment in this study.

\section{Other measurements}

The body mass index (BMI) was calculated from anthropometric data, using the following formula: BMI = weight $(\mathrm{kg}) /$ height $^{2}\left(\mathrm{~m}^{2}\right)$. Medical history (hypertension, ischemic heart disease, dyslipidemia, diabetes mellitus, and stroke) and educational years were assessed using self-reported questionnaires. Depressive symptoms were assessed using the self-reported Center for Epidemiologic Studies Depression Scale (CES-D), with scores ranging from 0 to 60 , where higher scores indicated more severe depressive symptoms (24). These variables at baseline were used as covariates.

\section{Statistical analyses}

Means \pm SDs were calculated for continuous variables, and numbers and percentages $(\%)$ were calculated for categorical variables. To compare the baseline characteristics of participants with and without cognitive impairment at follow-up, $\chi 2$ tests or t-tests were performed.

The sex stratified tertiles (T1-T3) of PDCAAS for each regular meal were computed at baseline. To characterize the people with low PDCAAS, participants were divided into two groups-group T1 (low) and group T2-T3 (medium and high) — based on each PDCAAS calculated at breakfast, lunch, dinner, and for the total day. Multivariable logistic regression analyses were performed to estimate odds ratios (ORs) with $95 \%$ confidence intervals (CIs) of the low PDCAAS group for incidence of cognitive impairment after 4 years, using the medium and high PDCAAS group as reference. PDCAASs for the three regular meals were analyzed together as independent variables. The covariates in model 1 were sex, age, BMI, CES-D score, educational years ( $0-7$ years, 8-15 years, or $\geq 16$ years (25)), history of lifestyle diseases (hypertension, dyslipidemia, diabetes mellitus, stroke, and ischemic heart disease), and MMSE score at baseline. In model 2, energy intakes at breakfast, lunch, and dinner at baseline were added to the variables in model 1. In model 3, protein intakes at breakfast, lunch, and dinner at baseline were added to the variables in model 1 . The variables-age, sex, BMI, educational years, CES-D, and medical history of diseases-were treated as covariates because they have previously been reported to be factors associated with cognitive function (26).

If we observed an association between the PDCAAS for each meal and cognitive function, we compared the amino acid ratios for that meal, based on the requirement pattern of the PDCAAS, between the low PDCAAS group and medium and high PDCAAS group using t-tests, to investigate which amino acids were insufficient. Additionally, we compared food-group consumption (g) per 100-kcal intake for each meal in the low PDCAAS group and medium and high PDCAAS group using t-tests. Statistical Analysis System version 9.3 (SAS Institute, Cary, NC, USA) was used to perform the analyses, and statistical significance was indicated by two-sided $\mathrm{P}$ values $<0.05$.

\section{Results}

The mean follow-up period was $4.2 \pm 0.4$ years. The age range of participants at baseline was $60-83$ years. Table 1 displays the baseline characteristics of participants with and without cognitive impairment in the follow-up study. During follow-up, 145 (26.8\%) participants developed cognitive impairment. When comparing participants with and without cognitive impairment at follow-up, baseline breakfast PDCAAS and MMSE scores in participants with cognitive impairment were significantly lower than those without cognitive impairment. 
Table 1. Baseline characteristics of participants with and without cognitive impairment at follow-up

\begin{tabular}{|c|c|c|c|}
\hline & \multicolumn{2}{|c|}{$\begin{array}{l}\text { Cognitive impairment } \\
\text { at follow-up }\end{array}$} & \multirow[t]{2}{*}{ P value } \\
\hline & With $(n=145)$ & Without $(n=396)$ & \\
\hline Men, n (\%) & $78(53.8)$ & $178(44.9)$ & 0.068 \\
\hline Age, years, M (SD) & $69.5(5.9)$ & $67.7(5.6)$ & 0.001 \\
\hline BMI, kg/m², M (SD) & $23.1(2.7)$ & $22.9(2.8)$ & 0.649 \\
\hline CES-D, range: $0-60, M(S D)$ & $7.9(7.7)$ & $7.1(6.7)$ & 0.232 \\
\hline \multicolumn{4}{|l|}{ Educational years, $\mathrm{n}(\%)$} \\
\hline $0-7$ years & $2(1.4)$ & $4(1.0)$ & $0.056^{*}$ \\
\hline $8-15$ years & $134(92.4)$ & $338(85.4)$ & \\
\hline$\geq 16$ years & $9(6.2)$ & $54(13.6)$ & \\
\hline Hypertension, n (\%) & $59(40.7)$ & $146(36.9)$ & 0.417 \\
\hline Dyslipidemia, n (\%) & $35(24.1)$ & $101(25.5)$ & 0.745 \\
\hline Diabetes mellitus, n (\%) & $16(11.0)$ & $33(8.3)$ & 0.332 \\
\hline Stroke, $\mathrm{n}(\%)$ & $11(7.6)$ & $14(3.5)$ & 0.047 \\
\hline Ischemic heart disease, $\mathrm{n}(\%)$ & $14(9.7)$ & $30(7.6)$ & 0.433 \\
\hline MMSE, range: 0-30, M (SD) & $28.7(0.7)$ & $29.1(0.8)$ & $<0.001$ \\
\hline \multicolumn{4}{|l|}{ Energy intake, kcal, M (SD) } \\
\hline Breakfast & $525.1(147.6)$ & $515.4(137.0)$ & 0.474 \\
\hline Lunch & $585.9(163.3)$ & $577.0(133.6)$ & 0.515 \\
\hline Dinner & $740.2(220.5)$ & $723.5(189.4)$ & 0.385 \\
\hline \multicolumn{4}{|l|}{ Protein intake, g, M (SD) } \\
\hline Breakfast & $20.1(7.3)$ & $20.2(6.7)$ & 0.884 \\
\hline Lunch & $22.1(7.1)$ & $22.3(6.6)$ & 0.854 \\
\hline Dinner & $30.7(9.8)$ & $32.1(9.1)$ & 0.108 \\
\hline \multicolumn{4}{|l|}{ Fat intake, g, M (SD) } \\
\hline Breakfast & $13.7(6.7)$ & $14.3(6.7)$ & 0.355 \\
\hline Lunch & $14.6(6.4)$ & $14.7(6.2)$ & 0.869 \\
\hline Dinner & $19.4(8.4)$ & $19.3(7.6)$ & 0.879 \\
\hline \multicolumn{4}{|l|}{ Carbohydrate intake, g, M (SD) } \\
\hline Breakfast & $80.1(24.3)$ & $76.3(23.0)$ & 0.095 \\
\hline Lunch & $88.4(26.8)$ & $86.5(21.2)$ & 0.382 \\
\hline Dinner & $93.8(27.3)$ & $92.4(23.8)$ & 0.571 \\
\hline \multicolumn{4}{|l|}{ PDCAAS, range: 0-100, M (SD) } \\
\hline Breakfast & $81.2(13.8)$ & $84.2(12.5)$ & 0.021 \\
\hline Lunch & $75.4(15.7)$ & $75.9(15.4)$ & 0.767 \\
\hline Dinner & $82.2(10.0)$ & $83.8(11.0)$ & 0.277 \\
\hline
\end{tabular}

$P$ values were obtained using the $\chi 2$ test for categorical variables and t-test for continuous variables. ${ }^{*} \mathrm{P}$ value was obtained using the Fisher exact test; $\mathrm{M}$, mean; SD, standard deviation; BMI, body mass index; CES-D, The Center for Epidemiologic Studies Depression Scale; MMSE, Mini-Mental State Examination; PDCAAS, protein digestibility-corrected amino acid score.

The ranges for PDCAASs with daily total, breakfast, lunch, and dinner were as follows: (1) low PDCAAS group for males: 49.91-82.37, 35.97-81.41, 19.71-70.48, and 37.55-80.57 respectively; (2) low PDCAAS group for females: $60.81-82.25,12.75-80.21,11.77-70.01$, and 49.33-80.21, respectively; (3) medium and high PDCAAS group for males: 82.41-100.0, 81.44-100.0, 70.71-100.0, and 80.62-100.0, respectively; and (4) medium and high
PDCAAS group for females: 82.26-100.0, 80.46-100.0, 70.28-100.0, and 80.28-100.0, respectively. Figure 2 (a, b) shows the food consumption at breakfast in the low PDCAAS group and medium and high PDCAAS group according to the PDCAAS at breakfast. The consumption of cereal grains, sugars and sweeteners, and fat and oils in the low PDCAAS group was significantly higher than that in the medium and high PDCAAS group. The consumption of eggs, beans and legumes, fish and seafood, and dairy products in the low PDCAAS group was significantly lower than that in the medium and high PDCAAS group.

In the multivariable logistic regression analysis of the total daily PDCAAS, no significant associations were observed in any of the models (OR, 1.40; 95\% CI, 0.92-2.12 in model 1; OR, 1.41; 95\% CI, 0.92-2.15 in model 2; and OR, 1.36; 95\% CI, 0.89-2.09 in model 3). In the analysis of PDCAASs for three regular meals, the breakfast PDCAAS alone displayed significant association with incidence of cognitive impairment after 4 years when adjusted for protein intake (Table 2).

Supplemental Figure 1 illustrates amino acid ratios at breakfast based on the requirement patterns. Amino acid ratios of the nine essential amino acids were significantly different between the low PDCAAS group and medium and high PDCAAS group ( $\mathrm{P}<0.001$ in all). Furthermore, the ratios of lysine, threonine, and tryptophan in the low PDCAAS group did not follow the requirement pattern.

\section{Discussion}

This is the first longitudinal epidemiological study to investigate the association between dietary protein quality assessed by PDCAAS and the incidence of cognitive impairment in community-dwelling older adults. In this study, the total daily PDCAAS did not show a significant association with cognitive function. Nevertheless, low PDCAAS at breakfast was significantly associated with the incidence of cognitive impairment, when PDCAASs at lunch and dinner, as well as protein intake were simultaneously included in the model. Our findings suggest that to maintain cognitive function in older adults, it is necessary to pay attention to a lower PDCAAS at breakfast (i.e., diet with poor quality protein), regardless of their protein intake.

A lower PDCAAS indicates poor quality protein in the diet, namely, the nutritional value of amino acids is low in that diet (6). Thus, a diet with low PDCAAS may be insufficient in amino acids needed to maintain brain function, especially in those who eat a diet with low protein (3); in other words, a diet with high PDCAAS may be important when protein intake is low. A report using data from the National Health and Nutrition Survey in Japan has shown that protein intake at breakfast in older adults was low (27). As breakfast is the first meal after an overnight fast, it is considered the most important meal of the day from a physiological perspective that supplies 
Figure 2. Food consumption at breakfast in the low and medium and high groups according to the PDCAAS at breakfast

(a)

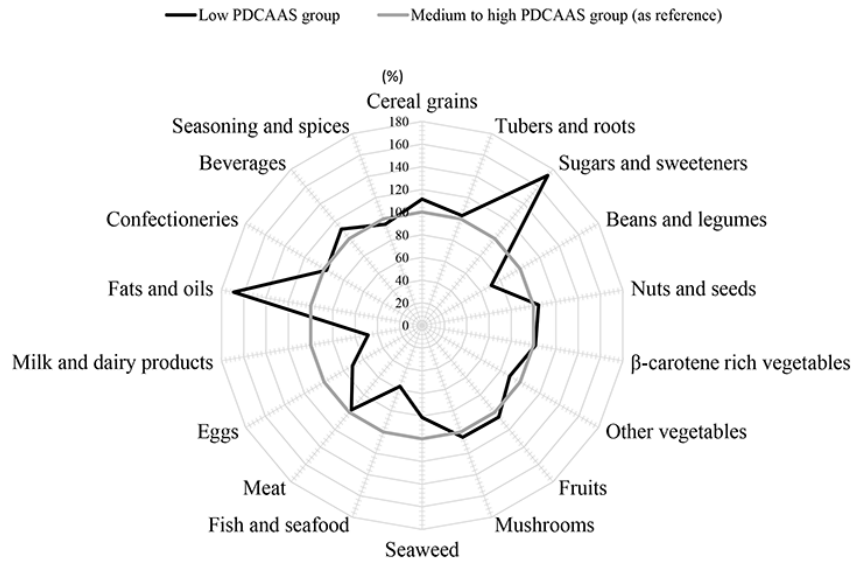

(b)
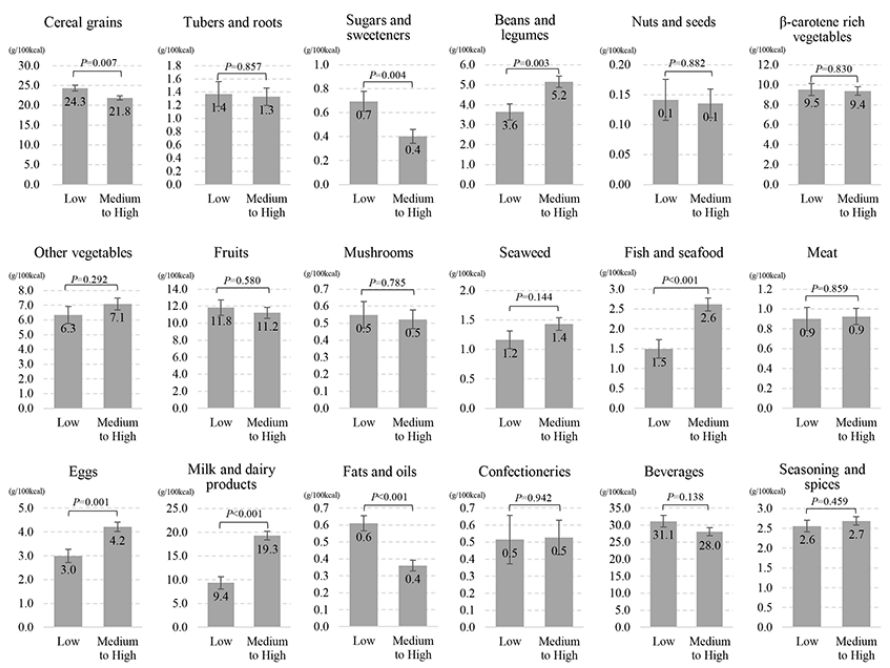

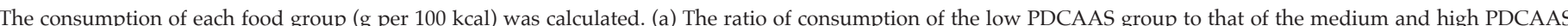

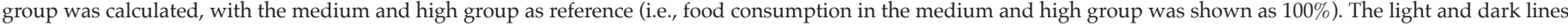

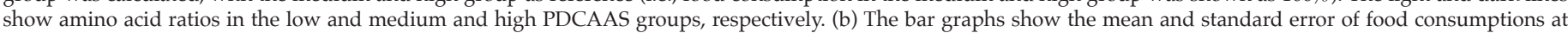

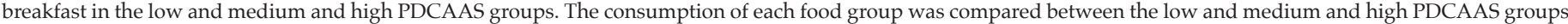
using a t-test; ${ }^{* *}, \mathrm{P}<0.001 ;{ }^{*}, \mathrm{P}<0.01$. PDCAAS, protein digestibility-corrected amino acid score.

energy and nutrients for working and learning activities $(28,29)$. Although, there have been no studies that investigated the association between meal composition of breakfast and cognitive function in older adults, studies on young to middle-aged people have demonstrated a significant association between breakfast content and cognitive function $(30,31)$. A research on healthy male students revealed that performances of working memory and peripheral attention were more accurate after intake of a high-protein breakfast than after a high-carbohydrate breakfast (30). A study on Chinese white-collar workers indicated that breakfast with adequate protein, according to the Chinese Dietary Guidelines, significantly improved cognitive function compared with that with inadequate protein (31). Thus, a diet with good quality protein, namely having a good nutritional value of amino acids, maybe more important at breakfast than at lunch or dinner.

On comparing the food consumptions at breakfast, we observed a lower intake of beans and legumes, milk and dairy products, fish and seafood, and eggs, and a higher intake of cereal grains, sugars and sweeteners, and fats and oils in the low PDCAAS group than in the medium and high PDCAAS group. Sugars and sweeteners, and fats and oils do not contain amino acids. Cereal grains have low nutritional content of amino acids and are deficient in lysine (19). Furthermore, amino acid ratios of lysine, threonine, and tryptophan in the low PDCAAS group were not sufficient for requirement patterns (Supplemental Figure 1). Low intake foods in the low PDCAAS group (i.e., beans and legumes, milk and dairy products, fish and seafood, and eggs) contain 1.1-1.5 times higher ratios of lysine, threonine, and tryptophan than their requirement patterns (19). Meanwhile, cereal grains that were highly consumed in the low PDCAAS group contain 0.3-0.6 times lower ratios of lysine; especially wheat, which has lower threonine and tryptophan, in addition to lysine (19). Therefore, eating a combination of various foods (e.g., cereal grain and fish) could improve the PDCAAS of the diet, i.e.,increase the protein quality. Good protein quality refers to the higher bioavailability of dietary protein (4). It is well known that excessive intakes of cereal grains and sugars and sweeteners lead to diabetes mellitus, which is a strong risk factor for dementia $(26,32)$. Thus, preference for sugar intake rather than protein quality might be associated with the occurrence of cognitive impairment.

This study has some limitations. First, we selected participants who could participate in both baseline and follow-up studies. Thus, there may be a bias in the study participant selection, with the inclusion of healthier people who could continue participating in the NILS-LSA survey. However, the food intake of the participants of this study was similar to that reported in the Japanese National Health and Nutrition Survey (33), which reflects the actual food intake among the Japanese population. Therefore, we believe that our results can be generalized to the older Japanese adult population. Second, we assessed the PDCAAS from the baseline dietary records alone, and dietary habits can change due to age-related factors (34). Further, although the PDCAAS might be a surrogate of many life-style factors which affect food intake, such as economic status, household situation, region, dietary culture, and so on, we did not assess these life-style factors. Thus, it might be unclear whether there is a causal relationship between the low 
Table 2. Multivariable-adjusted associations between PDCAAS at three regular meals and the incidence of cognitive impairment after 4 years $(n=541)$

\begin{tabular}{|c|c|c|c|c|c|c|}
\hline & \multicolumn{2}{|c|}{ Model 1} & \multicolumn{2}{|c|}{ Model 2} & \multicolumn{2}{|c|}{ Model 3} \\
\hline & OR $(95 \% \mathrm{CI})$ & $P$ value & OR $(95 \% \mathrm{CI})$ & $P$ value & OR $(95 \% \mathrm{CI})$ & $P$ value \\
\hline Breakfast PDCAAS (low group) & $1.55(1.03-2.35)$ & 0.038 & $1.57(1.02-2.40)$ & 0.039 & $1.58(1.00-2.50)$ & 0.048 \\
\hline Lunch PDCAAS (low group) & $0.86(0.56-1.32)$ & 0.481 & $0.87(0.56-1.34)$ & 0.517 & $0.85(0.54-1.34)$ & 0.495 \\
\hline Dinner PDCAAS (low group) & $1.11(0.73-1.69)$ & 0.636 & $1.12(0.73-1.72)$ & 0.594 & $1.08(0.71-1.65)$ & 0.717 \\
\hline Sex (Male) & $1.62(1.07-2.46)$ & 0.023 & $1.35(0.82-2.25)$ & 0.240 & $1.86(1.16-2.98)$ & 0.010 \\
\hline Age & $1.05(1.02-1.09)$ & 0.005 & $1.06(1.02-1.10)$ & 0.003 & $1.05(1.01-1.09)$ & 0.015 \\
\hline BMI & $1.02(0.95-1.10)$ & 0.612 & $1.02(0.94-1.10)$ & 0.663 & $1.02(0.95-1.10)$ & 0.556 \\
\hline CES-D & $1.01(0.98-1.04)$ & 0.502 & $1.01(0.98-1.04)$ & 0.478 & $1.01(0.98-1.04)$ & 0.542 \\
\hline Educational years & $0.49(0.24-0.96)$ & 0.039 & $0.50(0.25-0.99)$ & 0.045 & $0.47(0.24-0.93)$ & 0.031 \\
\hline Hypertension & $1.06(0.69-1.62)$ & 0.805 & $1.06(0.69-1.62)$ & 0.802 & $1.07(0.70-1.64)$ & 0.763 \\
\hline Ischemic heart disease & $1.11(0.54-2.29)$ & 0.778 & $1.16(0.56-2.40)$ & 0.698 & $1.06(0.51-2.21)$ & 0.883 \\
\hline Dyslipidemia & $0.99(0.61-1.60)$ & 0.965 & $1.01(0.62-1.63)$ & 0.978 & $0.98(0.61-1.59)$ & 0.943 \\
\hline Diabetes mellitus & $1.27(0.66-2.47)$ & 0.476 & $1.33(0.68-2.59)$ & 0.405 & $1.21(0.62-2.36)$ & 0.585 \\
\hline Stroke & $1.62(0.68-3.87)$ & 0.274 & $1.58(0.66-3.78)$ & 0.302 & $1.65(0.69-3.94)$ & 0.262 \\
\hline MMSE & $0.60(0.46-0.78)$ & $<0.001$ & $0.59(0.45-0.77)$ & $<0.001$ & $0.61(0.47-0.79)$ & $<0.001$ \\
\hline Energy intake at breakfast & - & - & $1.00(1.00-1.00)$ & 0.675 & - & - \\
\hline Energy intake at lunch & - & - & $1.00(1.00-1.00)$ & 0.742 & - & - \\
\hline Energy intake at dinner & - & - & $1.00(1.00-1.00)$ & 0.351 & - & - \\
\hline Protein intake at breakfast & - & - & - & - & $1.00(0.97-1.04)$ & 0.820 \\
\hline Protein intake at lunch & - & - & - & - & $1.00(0.97-1.04)$ & 0.944 \\
\hline Protein intake at dinner & - & - & - & - & $0.98(0.96-1.01)$ & 0.117 \\
\hline
\end{tabular}

Logistic regression analysis, medium and high PDCAAS group as reference. Model 1: includes explanatory variables as follows: PDCAAS at three regular meals (low/

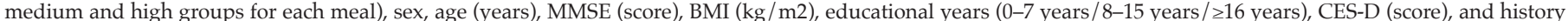

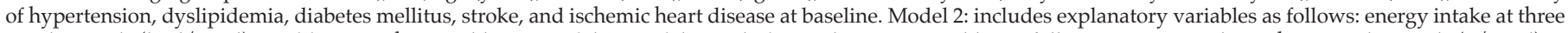

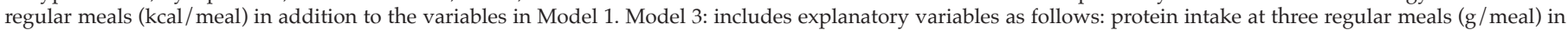

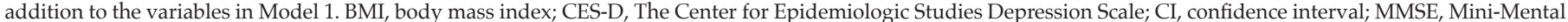
State Examination; OR, odds ratio; PDCAAS, protein digestibility-corrected amino acid score.

PDCAAS and occurrence of cognitive impairment. Third, we could not make a definitive diagnosis of dementia in our study because the cognitive function was assessed with the MMSE score alone. However, assessment of the self-reported medical history revealed that none of the participants reported dementia at baseline.

In conclusion, although there was no significant association between the PDCAAS of the total daily dietary intake and cognitive function, a lower PDCAAS of breakfast, i.e., a diet with poor quality of protein, was associated with the incidence of cognitive impairment in older adults of the community; thus, a diet with good protein quality, obtained by combining various foods to increase the PDCAAS, may be important for maintaining cognitive function in older adults.

Funding: This study was supported in part by grants from Ajinomoto Co. Inc. (Rei Otsuka, Kaori Kinoshita, and Hidenori Arai, grant number none) and Research Funding for Longevity Sciences from the National Center for Geriatrics and Gerontology, Japan (Rei Otsuka, grant number 19-10, 21-18). The sponsors had no role in the design and conduct of the study; in the collection, analysis, and interpretation of data; in the preparation of the manuscript; or in the review or approval of the manuscript.
Acknowledgments: We would like to thank all participants and staff in the NILSLSA for their cooperation and contribution to this study. We would also like to thank Tomonori Koizumi at Ajinomoto Co., Inc. for support and advice regarding the calculation methods for the amino acid scores. We would like to thank Editage (www.editage.com) for English language editing.

Conflict of Interest Disclosure: Michihiro Takada, Masako Tsukamoto-Yasui, Hiroko Jinzu, and Akira Imaizumi are employees of Ajinomoto Co., Inc. Kaori Kinoshita, Rei Otsuka, and Hidenori Arai received grants from Ajinomoto Co. Inc. Kaori Kinoshita, Rei Otsuka, Michihiro Takada, Masako Tsukamoto-Yasui, Hiroko Jinzu, Akira Imaizumi, and Hidenori Arai have applied for patents for "food evaluation methods for cognitive function." There were no financial, professional, and personal relationships with potential to bias the work. Support from Ajinomoto Co., Inc. included only direct financial support and no support in kind, such as provision of equipment without charge or at reduced cost, and provision of services, such as statistical analysis.

Ethical Standards: This study was carried out in accordance with the appropriate ethical standards.

Author Contributions: Kaori Kinoshita conceived the study design, performed the data analysis, interpreted the results, and drafted the initial manuscript; Rei Otsuka collected data, conceived the study design, interpreted the results, contributed to discussions, and had primary responsibility for the final content; Michihiro Takada conceived the study design, interpreted the results, and contributed to discussions; Masako Tsukamoto-Yasui conceived the study design; Yukiko Nishita, Chikako Tange, and Makiko Tomida collected data, interpreted the results, and contributed to discussions; Hiroko Jinzu interpreted the results and contributed to discussions; Hiroshi Shimokata designed the NILS-LSA, interpreted the results, and contributed to discussions; Masafumi Kuzuya supervised the study, interpreted the results, and contributed to discussions; Akira Imaizumi conceived the study design and interpreted the results; Hidenori Arai supervised 
the study, conceived the study design, interpreted the results, and contributed to discussions. All authors critically revised the manuscript for intellectual content and approved the final manuscript. All authors agree to be accountable for all aspects of the work in ensuring that questions related to the accuracy or integrity of any part of the work are appropriately investigated and resolved.

Open Access: This article is distributed under the terms of the Creative Commons Attribution 4.0 International License (http:/ / creativecommons.org/ licenses/by/4.0/), which permits use, duplication, adaptation, distribution and reproduction in any medium or format, as long as you give appropriate credit to the original author(s) and the source, provide a link to the Creative Commons license and indicate if changes were made.

\section{References}

1. Giudici KV. Nutrition-Based Approaches in Clinical Trials Targeting Cognitive Function: Highlights of the CTAD 2020. J Prev Alzheimers Dis 2021;8:118-122.

2. Glenn JM, Madero EN, Bott NT. Dietary protein and amino acid intake: links to the maintenance of cognitive health. Nutrients 2019;11(6):1315.

3. van de Rest O, van der Zwaluw NL, de Groot LC. Literature review on the role of dietary protein and amino acids in cognitive functioning and cognitive decline. Amino Acids 2013;45(5):1035-1045.

4. World Health Organization. Protein and amino acid requirements in human nutrition: report of a joint WHO/FAO/UNU expert consultation. WHO Technical Report Series No. 935. 2007. Geneva, Switzerland

5. World Health Organization. Energy and protein requirements: report of a joint FAO/WHO/UNU expert consultation. WHO Technical Report Series No. 724. 1985. Geneva, Switzerland.

6. Food and Agriculture Organization of the United Nations. Protein quality evaluation: report of the joint FAO/WHO expert consultation. FAO Food and Nutrition Paper No. 51. 1991. Rome, Italy

7. Food and Agriculture Organization of the United Nations. Dietary protein quality evaluation in human nutrition: report of an FAO expert consultation FAO Food and Nutrition Paper No. 92. 2013. Rome, Italy

8. Lee WT, Weisell R, Albert J, et al. Research approaches and methods for evaluating the protein quality of human foods proposed by an FAO expert working group in 2014. J Nutr 2016;146(5):929-932.

9. Schaafsma G. Advantages and limitations of the protein digestibility-corrected amino acid score (PDCAAS) as a method for evaluating protein quality in human diets. Br J Nutr 2012;108(Suppl 2):S333-S336.

10. Otsuka R, Kato Y, Nishita Y, et al. Age-related changes in energy intake and weight in community-dwelling middle-aged and elderly Japanese. J Nutr Health Aging 2016;20(4):383-390.

11. Bauer J, Biolo G, Cederholm T, et al. Evidence-based recommendations for optimal dietary protein intake in older people: a position paper from the PROT-AGE Study Group. J Am Med Dir Assoc 2013;14(8):542-559.

12. Pot GK, Almoosawi S, Stephen AM. Meal irregularity and cardiometabolic consequences: results from observational and intervention studies. Proc Nutr Soc 2016;75(4):475-486.

13. Shimokata H, Ando F, Niino N. A new comprehensive study on aging - the National Institute for Longevity Sciences, Longitudinal Study of Aging (NILSLSA). J Epidemiol 2000;10(Suppl 1):S1-S9.

14. Matthews D. BSE: a global update. J Appl Microbiol 2003;94(Suppl):120S-125S.

15. Tariq SH, Tumosa N, Chibnall JT, Perry MH 3rd, Morley JE. Comparison of the Saint Louis University mental status examination and the mini-mental state examination for detecting dementia and mild neurocognitive disorder - a pilot study. Am J Geriatr Psychiatry 2006;14(11):900-910.
16. Saxton J, Morrow L, Eschman A, Archer G, Luther J, Zuccolotto A. Computer assessment of mild cognitive impairment. Postgrad Med 2009;121(2):177-185.

17. Kaufer DI, Williams CS, Braaten AJ, Gill K, Zimmerman S, Sloane PD. Cognitive screening for dementia and mild cognitive impairment in assisted living: comparison of 3 tests. J Am Med Dir Assoc 2008;9(8):586-593.

18. Imai T, Sakai S, Mori K, Ando F, Niino N, Shimokata H. Nutritional assessments of 3-day dietary records in National Institute for Longevity Sciences - Longitudinal Study of Aging (NILS-LSA). J Epidemiol 2000;10(Suppl 1):S70-S76.

19. Ministry of Education, Culture, Sports, Science and Technology-Japan. Standard tables of food composition in Japan. (Seventh revised version). 2015. https: / / www.mext.go.jp/en/policy/science_technology/policy/title01 / detail01/1374030.htm. Accessed 20 January 2021

20. Kaneko K, Ishikawa K, Setoguchi K, Koike G. Utilization and requirement of dietary protein taking into account the dermal and miscellaneous nitrogen losses in Japanese women. J Nutr Sci Vitaminol (Tokyo) 1988;34(5):459-467.

21. Mori E, Mitani Y, Yamadori A. Usefulness of a Japanese version of the minimental state test in neurological patients. Jpn J Neuropsychol 1985;1:82-90.

22. Folstein MF, Folstein SE, McHugh PR. "Mini-mental state". A practical method for grading the cognitive state of patients for the clinician. J Psychiatr Res 1975;12(3):189-198.

23. Mitchell AJ. A meta-analysis of the accuracy of the mini-mental state examination in the detection of dementia and mild cognitive impairment. J Psychiatr Res 2009;43(4):411-431.

24. Radloff LS. The CES-D Scale: A self-report depression scale for research in the general population. Appl Psychol Meas 1977;1(30):385-401.

25. Petersen RC, Aisen PS, Beckett LA, et al. Alzheimer's Disease Neuroimaging Initiative (ADNI): clinical characterization. Neurology 2010;74(3):201-209.

26. Livingston G, Sommerlad A, Orgeta V, et al. Dementia prevention, intervention, and care. Lancet 2017;390(10113):2673-2734.

27. Jenkins DJ, Wolever TM, Taylor RH, et al. Slow release dietary carbohydrate improves second meal tolerance. Am J Clin Nutr 1982;35(6):1339-1346.

28. Xu J, Begley P, Church SJ, et al. Graded perturbations of metabolism in multiple regions of human brain in Alzheimer's disease: Snapshot of a pervasive metabolic disorder. Biochim Biophys Acta 2016;1862(6):1084-1092.

29. O'Sullivan TA, Robinson M, Kendall GE, et al. A good-quality breakfast is associated with better mental health in adolescence. Public Health Nutr 2009;12(2):249-258.

30. Fischer K, Colombani PC, Langhans W, Wenk C. Carbohydrate to protein ratio in food and cognitive performance in the morning. Physiol Behav 2002;75(3):411-423.

31. Tang Z, Zhang N, Liu A, et al. The effects of breakfast on short-term cognitive function among Chinese white-collar workers: protocol for a three-phase crossover study. BMC Public Health 2017;17(1):92.

32. Sinclair AJ, Vellas B. Diabetes Mellitus and Cognitive Decline - Prevention Should Not Be Delayed! J Prev Alzheimers Dis 2018;5:95-97.

33. Ministry of Health, Labour and Welfare National Health and Nutrition Survey. http: / / www.nibiohn.go.jp/eiken/english/research/project_nhns. html. Accessed 22 January 2021

34. Zhu K, Devine A, Suleska A, et al. Adequacy and change in nutrient and food intakes with aging in a seven-year cohort study in elderly women. J Nutr Health Aging 2010;14(9):723-729.

How to cite this article: K. Kinoshita, R. Otsuka, M. Takada, et al. Low Amino Acid Score of Breakfast is Associated with the Incidence of Cognitive Impairment in Older Japanese Adults: A Community-Based Longitudinal Study J Prev Alz Dis 2022;1(9):151-157; http: / / dx.doi.org/10.14283/jpad.2021.25 